\title{
Ciliary Neurotrophic Factor (CNTF) affects the excitable and contractile properties of innervated skeletal muscles
}

\author{
BEATRIZ U. RAMIREZ ${ }^{1}$, LEIRA RETAMAL ${ }^{2}$ and CECILIA VERGARA ${ }^{2}$ \\ ${ }^{1}$ Facultad de Ciencias Médicas, USACH \\ ${ }^{2}$ Departamento de Biología, Facultad de Ciencias, U. de Chile
}

\begin{abstract}
The well-established trophic role of CNTF upon neurons led to performing clinical trials in patients of neurodegenerative diseases. However, trials were suspended due to side effects such as severe weight loss, hyperalgesia, coughing, muscle cramps and pain. So far it is not known how CNTF triggers the problems related to skeletal muscle cramps and pain. CNTF has also been described as a myotrophic factor for denervated skeletal muscles, but the possibility that it affects innervated muscles has also been considered. Since a myotrophic factor could be a valuable tool for treatment of several muscle diseases, we studied the effects of low doses of CNTF delivered systemically by an osmotic pump, over the electrical and mechanical properties of innervated and denervated fast and slow muscles.

CNTF induced spontaneous electrical discharges and slowed twitches in innervated muscles, but did not prevent the changes induced by denervation. We postulate that the spontaneous discharges induced by CNTF in innervated muscles may be the cause of the cramps, coughing, and muscle ache reported by patients. At low doses, CNTF does not exert its myotrophic role over denervated muscles but clearly affects the excitable and contractile properties of innervated muscles.
\end{abstract}

Key terms: CNTF, contraction and relaxation time, denervation, EMG, fibrillation, skeletal muscle.

\section{INTRODUCTION}

Ciliary neurotrophic factor (CNTF), a cytokine expressed by glial cells, acts as a survival factor for motor and sensory neurons and that has also been described as having myotrophic properties (Helgren et al. 1994, Sleeman et al. 2000, Huang et al 2002). The receptor for CNTF, present in neurons and skeletal muscles, is an heterotrimer formed by a specific CNTF receptor subunit $(\mathrm{CNTFR} \alpha)$, the beta subunit of the leukemia inhibitory factor (LIFRB) and glycoprotein gp130.

After the success of CNTF in pre-clinical trials this cytokin has been used in patients with neurodegenerative diseases, such as amyotrophic lateral sclerosis (ALS) and is being considered for use in Huntington's disease (HD) to prevent or cure neuronal degeneration and their associated motor and mental problems (ALS Study Group
1996, Bachoud-Levi 2000, Kordower 1999).

The initial clinical trials with ALS patients used peripheral subcutaneous administration of CNTF, but patients reported severe side effects that included anorexia, skeletal muscle weight loss, hyperalgesia, coughing that was not related to lung problems, cramps and muscle pain (ALS Study Group 1996, Windisch 1998). As a way to circumvent these side effects, later studies have used intrathecallydelivered CNTF. With this application method one suspects that the peripheral circulating levels of CNTF would be quite low and patients would not suffer from anorexia or skeletal muscle weight loss, although they did report muscle cramps and pain (Penn et al. 1997).

On the other hand, in the peripheral nervous system, CNTF is found in high quantities in Schwann cells around the 
sciatic nerve and upon denervation, circulating CNTF increases and also the specific CNTF receptor binding alfa subunit (CNTFR- $\alpha$ ) increases in skeletal muscle (Weis et al. 1998).

Denervation induces many changes in the muscle cells. Among the most obvious are atrophy, the appearance of fibrillatory potentials, slowing of twitch kinetics and altered expression levels of many proteins, included CNTFR $\alpha$. Helgren et al. (1994) proposed that $\mathrm{CNTF}$ has a myotrophic role because they found that this cytokine attenuates the skeletal muscle wasting induced by transection of the sciatic nerve and also spares the slowing of the twitch (Helgren et al. 1994). Nevertheless, Martin et al. (1996), using similar doses of CNTF, reported that CNTF did not protect denervated muscles, but caused wasting of normally-innervated fast and slow muscles.

We have attempted to understand the basis of the controversial reports concerning the myotrophic effects of CNTF on rat muscle and the cause of side effects reported by patients, even at low plasmatic concentrations of CNTF.

To this aim we used rats as a model to study the effects of chronic systemic application of low doses of CNTF over: a) skeletal muscle contractile and electrical properties in both, innervated and denervated preparations, and b) lower leg muscle weight. Because a possible crosstalk between the signal transduction pathways mediated by cAMP and the CNTF-mediated signaling has been described in some cell types (McLennan, 1996), we also tested the effect of forskolin, an activator of adenylate cyclase.

We found that at low doses, CNTF induces the appearance of spontaneous electrical discharges in innervated muscles. It also induced a slowing of the twitch, only in innervated muscles, and did not protect the muscles from the denervation-induced lengthening of the twitch. The spontaneous electrical activity could account for the cramps or pain described by patients treated with this cytokine. Forskolin also affected the kinetic parameters of the twitch only for innervated muscles. Doses of CNTF that induced spontaneous potentials did not affect the weight of either innervated or denervated muscles.

\section{METHODS}

Male Sprague-Dawley rats aged 40 days were anaesthetized with an i.p. injection of a ketamine $(80 \mathrm{mg} / \mathrm{Kg})$ and xylazine hydrochloride (12 $\mathrm{mg} / \mathrm{Kg})$ mixture. Animals were divided into 1 control and 4 experimental groups. Control animals (controls) received the osmotic pump loaded with saline. Animals in the first experimental group (unilateral denervation) were subjected to unilateral sciatic nerve section at the ischiatic notch height, (3-5 $\mathrm{mm}$ of the nerve removed). Animals in the second and third groups were unilaterally denervated as described and received a pump loaded with $0.5 \mu \mathrm{g} / \mathrm{ml} \operatorname{rrCNTF}$ (R\&D Systems) dissolved in phosphate buffered saline containing $0.1 \%$ bovine serum albumin (unilateral denervation + CNTF) or loaded with $100 \mu \mathrm{M}$ forskolin (Calbiochem) (unilateral denervation + forskolin). In the fourth experimental group, normal animals, whose sciatic nerves were untouched, received a pump loaded with CNTF (normals + CNTF). The pump (Alzet 2002) was implanted in a pocket under the skin in the back of the animals and delivered $0.5 \mu \mathrm{l} / \mathrm{hr}$. The drug or saline was distributed through the systemic circulation to the target tissues because no catheter was attached to the pump. The stability and bioactivity of CNTF delivered through these pumps has been established previously by the work of Guillet et al. 1999.

All rats received (i.p.) $0.1 \mathrm{ml}$ of $0.5 \%$ Baytril (Bayer) to prevent infections. The animals were left to recover for 10 days with free access to food and water. Each animal was weighed before the operation as well as 10 days after, before the electromyogram (EMG) and tension recordings were performed.

\section{Electromyogram (EMG) recording}

The tibialis anterior (TA) EMG was recorded in anaesthetized animals with a 
concentric electromyography electrode (Nicolet 019-721300, gauge 27) inserted into the muscle belly close to the knee or close to the ankle. The electrode was connected into a Power Lab $4 \mathrm{~s}$ data acquisition system. Electrical activity was recorded at a sampling rate of $1 \mathrm{KHz}$. A high pass filter was set at $10 \mathrm{~Hz}$ and a low pass filter at $500 \mathrm{~Hz}$. The input amplifier was connected to a loudspeaker in order to hear the discharges. Occasionally the EMG from the extensor digitorum longus (EDL), soleus or gastrocnemius muscles was also recorded. The intensity of the electrical activity was quantified as the standard deviation of $3 \mathrm{~s}$ of recording, measured in $\mu \mathrm{V}$. The baseline recorded in a resting muscle at high amplification shows a very narrow range of potential (low noise), and therefore a small standard deviation. The presence of variable amplitude potentials over the baseline introduces a large variability and therefore a higher standard deviation. The statistical analys is to determine whether recordings from different experimental conditions were significantly different or not was done at a fixed time after inserting the recording electrode.

The denervation of the muscles was checked before recording by the lack of response upon central stimulation of the nerve.

\section{Tension recordings}

Tension was recorded in vivo at room temperature $\left(\sim 20^{\circ} \mathrm{C}\right)$ after isolating the distal tendon of the slow soleus and the fast EDL. The leg was fixed to a speciallydesigned table to hold the rat leg with two fixtures, one inserted at the knee and one at the ankle. Each tendon was tied to a semiisometric force transducer (UFI, model 1030), taking care that the thread was at a right angle to the transducer. Basal muscle length was adjusted for maximal tension. The transducer was connected to the acquisition system (Power Lab 4s). Data were recorded at $1 \mathrm{KHz}$. Twitch kinetic parameters were determined after direct supramaximal stimulation of the muscle.
After recordings, gastrocnemius, soleus, EDL and TA muscles were dissected and frozen in liquid nitrogen into pre-weighted Eppendorf tubes. The weight of each muscle was determined later.

\section{Statistical analysis}

Results are expressed as mean \pm SEM. The measurements were made on the number of animals indicated in the corresponding figure, table or text. Statistical significance of the results was tested by the Student's " $t$ " test.

\section{RESULTS}

Effects of CNTF and forskolin over the excitability of the nerve-muscle system. In the presence of CNTF, innervated muscles developed spontaneous potentials.

The EMG of control animals was very reproducible, there was just a small baseline noise and no spontaneous activity after insertional activity had vanished. The EMG recorded from the normal + CNTF treated rats showed spontaneous potentials that differed from fibrillation in sound and frequency. In most muscles we recorded small $(20 \mu \mathrm{V})$ isolated spikes, but frequently we recorded bursts of spontaneous potentials with large spikes of approximately $200 \mu \mathrm{V}$, as shown in Figure 1. The amplitude of these spontaneous potentials varied among different normal + CNTF treated muscles, but because they occurred over a stable base line, they were readily distinguishable from normal EMGs. Similar activity was recorded for the innervated muscles of unilaterally denervated + CNTF treated animals. Therefore, data from muscles of normal + CNTF treated animals and from the innervated muscles of unilaterally denervated + CNTF treated animals were pooled for statistical analysis of the membrane potentials after CNTF treatment. Because statistical analysis of the intensity of the membrane potentials was done over three seconds of recording at a fixed time 


\section{Innervated}
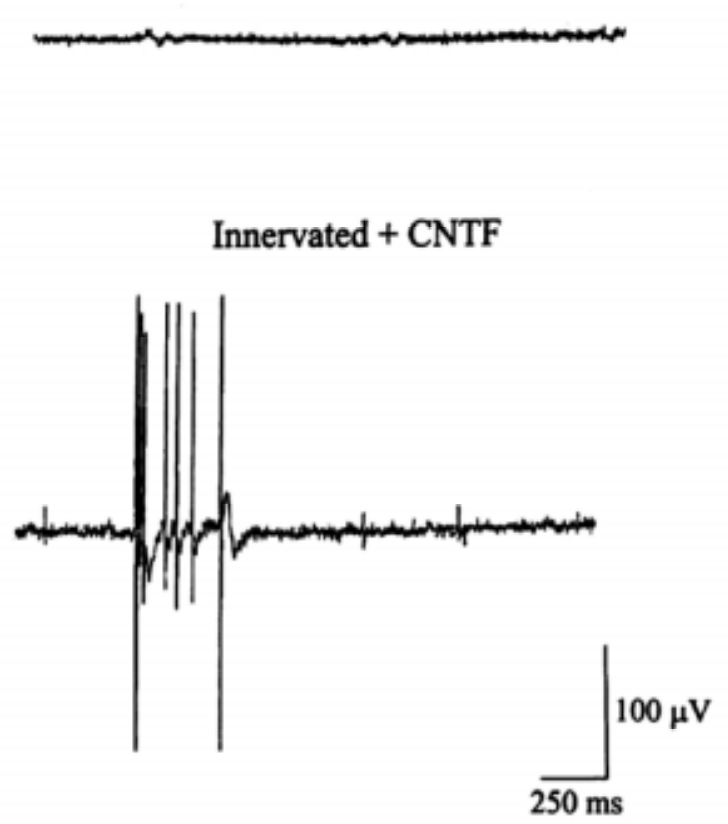

Figure 1. CNTF induces spontaneous discharges in innervated muscles. EMG recordings from the innervated Tibialis Anterior of a normal control rat and from an animal that was exposed to CNTF for 10 days. The spontaneous activity recorded from the innervated CNTF-treated muscle is different from fibrillation and shows a burst of high amplitude spikes and a few low amplitude potentials.

after inserting the electrode, the evaluated period for each condition was not necessarily the one with the highest activity for each recording. Normal muscles displayed a low basal activity, accounted for only by noise. As shown in Table I, the spontaneous bursts induced by CNTF result in a doubling of the value for standard deviation in the innervated TA muscles, as compared with normal (untreated) muscles. Innervated EDL developed similar spontaneous electrical discharges after CNTF treatment (data not shown). The spontaneous potentials developed in CNTFexposed innervated muscles were intense enough to trigger spontaneous contractions. Figure 2 shows a spontaneous twitch that appeared after a twitch induced by electrical stimulation of a normal + CNTF treated EDL muscle. The spontaneous twitch was smaller than the evoked one, indicating that only a fraction of the total number of muscle fibers were contracting at the same time. The occurrence of spontaneous twitches is an extremely rare phenomena in a normal neuromuscular preparation, and we never recorded one in any of the control animals.

EMG recordings from innervated muscles of forskolin- treated animals were identical to the controls.

Neither CNTF nor forskolin affect the fibrillatory potentials in denervated muscles

Figure 3 shows EMG recordings from control, denervated and denervated + CNTF muscles. The EMG was recorded in two

\section{TABLE I}

CNTF increases electrical activity only in innervated muscles

The intensity of electromyographic activity of Tibialis Anterior muscles was quantified as the standard deviation of 3 seconds of recording. After CNTF treatment this parameter doubled for innervated muscles, due to the presence of sporadic bursts superimposed over basal activity. The fibrillatory activity of denervated muscle results in a high value of standard deviation, as compared with normal innervated muscles, due to the big variability of the fibrillatory potentials. Nevertheless, this value is not statistically different for denervated only and denervated + CNTF muscles, therefore, we can not suggest that CNTF affects fibrillatory activity in denervated muscles.

\begin{tabular}{lcc}
\hline Condition & $\mu \mathrm{V}$ & $\mathrm{P}$ \\
\hline Normal innervated TA & $3.32 \pm 0.33(8)$ & $<0.001$ \\
Innervated + CNTF & $6.44 \pm 0.47(8)$ & n.s. \\
Denervated & $13.67 \pm 2.15(16)$ & \\
Denervated + CNTF & $17.96 \pm 2.14(16)$ & \\
\hline
\end{tabular}

Data are mean \pm SEM. In brackets, the number of independent records measured n.s: non-significant 


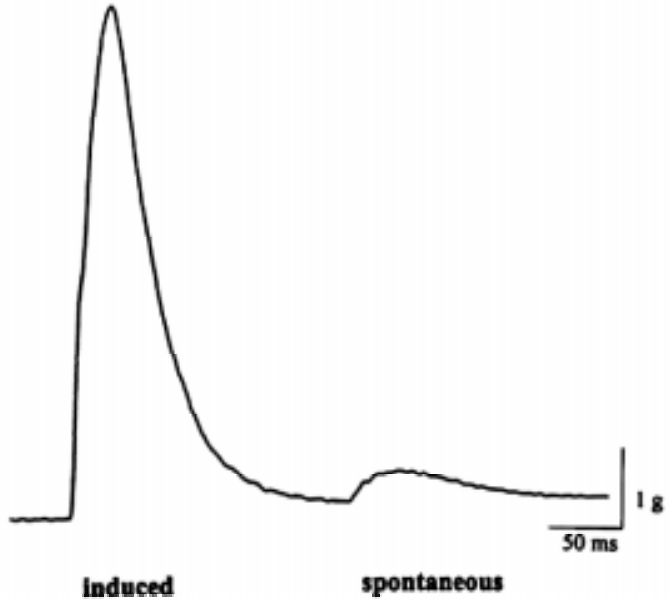

Figure 2. CNTF induces spontaneous contraction. Isometric tension recorded from an innervated EDL muscle previously exposed to CNTF for 10 days. The record shows a twitch induced by electrical stimulation followed by a spontaneous contraction.

different spots in every muscle (near the knee or the ankle) but no difference was detected between the two records in each muscle. As expected, denervation induced the appearance of fibrillatory potentials. Fibrillatory activity consisted of spontaneous potentials of variable amplitude and frequency displayed during the whole recording period, and therefore was quite different from the short bursts that occur in innervated muscles treated with CNTF. The frequency and intensity of fibrillatory potentials were quite variable among different animals for both denervated or denervated + CNTF muscles. As shown in Table I, there was no statistically-significant difference between the intensity of the electrical activity of only denervated and denervated + CNTF treated muscles. The same lack of effect on fibrillatory activity was observed in forskolin-treated animals (not shown).

Effects of CNTF and forskolin on the twitch kinetics for innervated or denervated EDL and soleus muscles

We evaluated the effects of CNTF and forskolin upon the twitch characteristics in the unilateral denervated animals treated with either drug, using the muscles from animals that had received unilateral section of the sciatic nerve, but no drug treatment as controls. The twitch parameters were evaluated in fast (EDL) and slow (soleus) muscles in both legs. As expected, in control animals, all denervated muscles had slower contraction and half-relaxation times than the corresponding contralateral innervated muscles (Fig 4, front and back columns at the left in each panel). Figure $4 \mathrm{~A}$ shows that in the presence of CNTF or forskolin, contraction time is significantly increased for the innervated soleus (front middle and right columns). Nevertheless, neither drug caused a significant diminution in contraction time for the denervated muscle (back middle and right columns). For the EDL (Fig 4C), the effects of these drugs are very similar, except that in this case CNTF potentiates the effect of

\section{Innervated}

\section{Denervated}

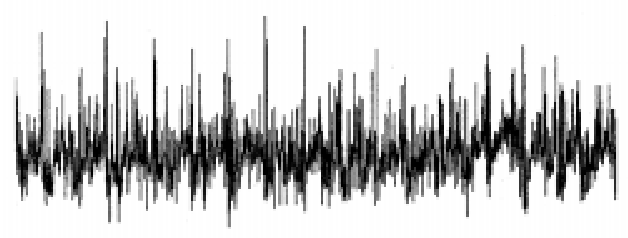

\section{Denervated + CNTF}

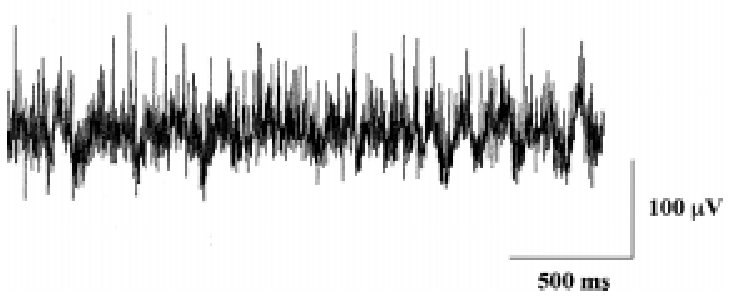

Figure 3. CNTF does not alter fibrillatory activity in denervated muscles. EMG recordings from the distal portion of the Tibialis Anterior. The upper record is from an innervated muscle, the second from a 10-day denervated muscle, and the third from a 10-day denervated-CNTF exposed muscle. Denervated muscles show typical fibrillatory potentials. 
denervation with respect to the slowing of contraction time (back left and middle columns). For the innervated EDL, CNTF increases contraction time from $36.6 \pm 0.8$ to $40.8 \pm 2.2$ and forskolin to $41.3 \pm 0.5$ (mean \pm SEM). Even though both drugs increase contraction time, the high dispersion of the data for CNTF-treated muscles makes the difference insignificant (Fig $4 \mathrm{C}$ front middle column). With respect to half-relaxation times, CNTF again caused a slowing of this parameter for innervated soleus and EDL muscles, but we did not find that either drug caused a diminution of the slowing caused by denervation (Fig 4B and D). For the EDL, CNTF also intensified the effect of denervation (Fig 4D, left and middle columns at the back). In summary, our studies showed that at low doses, CNTF did not counteract the denervation-induced slowing of the contractile characteristics of the twitch, but did induce a slowing of the twitch in innervated muscles (Fig 5) and also intensify the effect of denervation for the EDL. Because innervated muscles became slower, the difference between denervated and innervated muscles decreased.

Forskolin behaved as CNTF for contraction time and did not affect halfrelaxation time.

A low dose of CNTF does not affect the wet weight of normal or denervated skeletal muscles

The effect of CNTF on the weight of denervated and innervated lower leg muscles (TA, EDL, soleus and gastrocnemius) was studied in the animals that received unilateral sciatic nerve sectioning after 10 days of treatment. Controls were animals that received unilateral sciatic nerve transection but no CNTF. As expected, in control animals all the denervated muscles decreased in weight, as compared with the corresponding contralateral innervated muscles. The decrease was approximately $40 \%$ for the TA, soleus and gastrocnemius but only $\sim 25 \%$ for the EDL, consistent with the
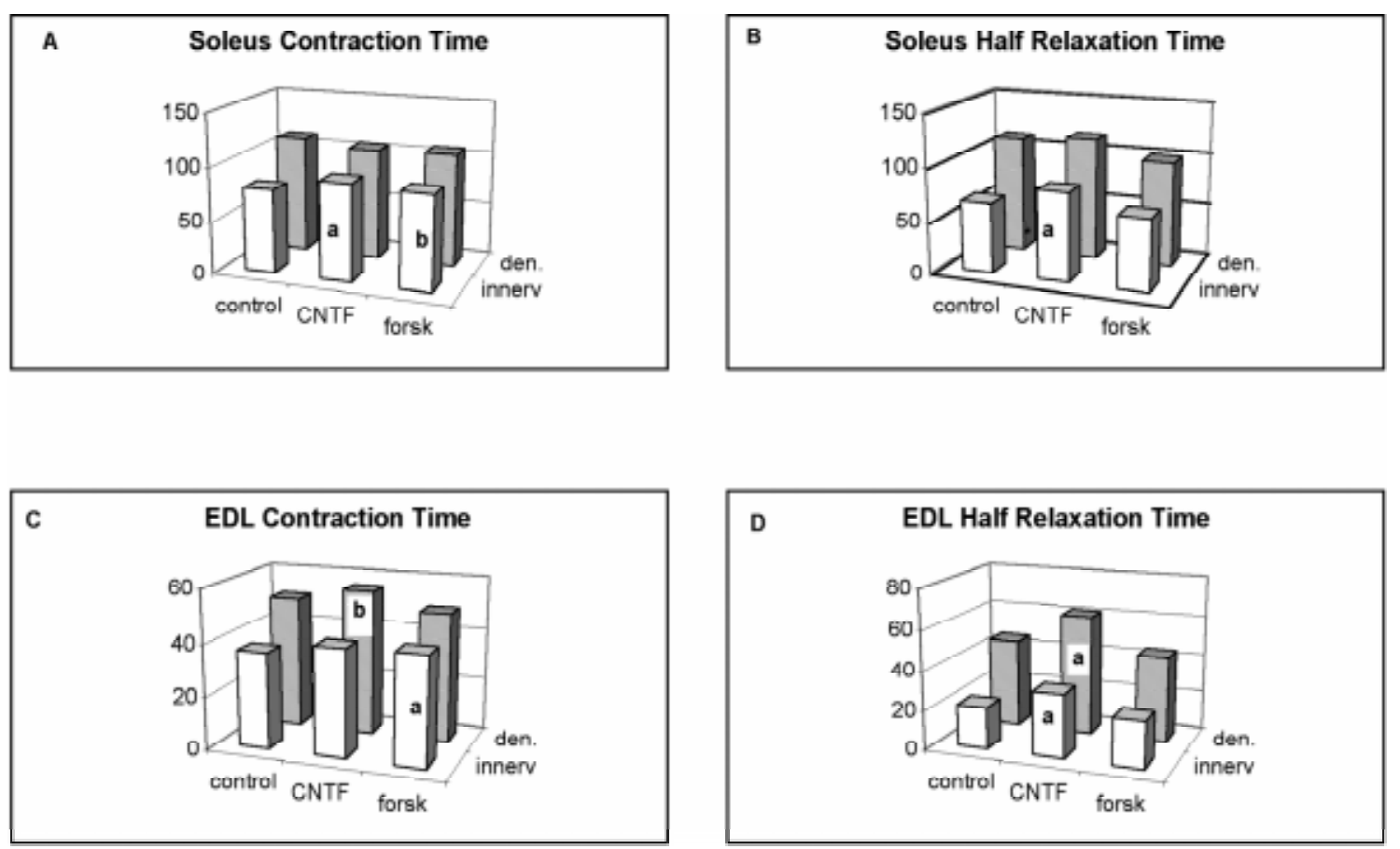

Figure 4. CNTF alters kinetic parameters of the twitch. Twitch kinetic parameters from control, CNTF or forskolin exposed animals. In all animals one sciatic nerve was cut. The figure shows the average for 6-9 controls and 5-9 drug-treated muscles for contraction and half-relaxation times. Statistically-significant differences were calculated separated for innervated or denervated muscles (Student's " $t$ "-test; $a=p<0.01, b=p<0.05$ ). In each case drug treatment was compared to its corresponding (innervated or denervated) control. The standard error for each condition is too small to be appreciated at the scale shown. 


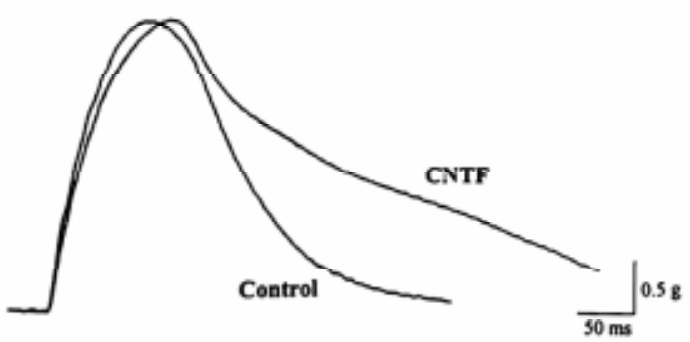

Figure 5. CNTF slows the twitch in innervated muscles. Isometric twitches evoked by stimulation of the soleus in a normal animal (control) and in a rat treated with CNTF for 10 days. Despite both muscles being innervated, the twitch from the CNTF-exposed muscle presents a slower time course.

slower time course of weight loss described for this muscle (Helgren et al., 1994). As shown in Table II, CNTF exposure had no effect on the weight of innervated or denervated muscles. The difference between muscles in the control group and the CNTF-treated animals was not significant for the four different muscles studied, whether innervated or denervated.

\section{DISCUSSION}

The most important finding of this study is that low doses of CNTF evoke spontaneous potentials in innervated skeletal muscles. Also, the kinetic parameters of the twitch of innervated muscles are altered by this cytokine.

The suggested use of CNTF to prevent neurodegeneration in HD and ALS (ALS Study Group 1996, Kordower et al., 1999) requires long-term application of this drug. Therefore, it is important to ascertain its effects not only on degenerating neurons, but also on other target tissues such as denervated and innervated muscles. The spontaneous electrical activity of innervated CNTF-exposed muscles reported here could be related to the leg and back cramps reported by CNTF-treated patients (Penn et al., 1997). In our hands, application of CNTF provoked small muscle contractions. A similar situation could have been described as cramps by patients treated with this cytokine.

To our knowledge, this is the first report showing that CNTF can induce spontaneous electrical discharges in innervated muscles. Although we do not know the mechanisms, this effect may result from: a) direct action of CNTF on the muscle fibers, b) indirect action mediated by the corresponding motoneuron, or c) indirect action of CNTF over blood born cells that could liberate other cytokines responsible for the observed effects.

Direct effects of any drug over the muscle should be observed over denervated as well as over innervated fibers. If only innervated muscles are affected the effect of the drug is probably nerve mediated.

\section{Differential effects of CNTF over innervated and denervated muscles}

We suspect that CNTF may have both direct and nerve-mediated effects on the twitch kinetic parameters. Direct effects on muscle

\section{TABLE II}

A low dose of CNTF does not affect weight for innervated or denervated muscles

Weight of lower leg muscles (Tibialis Anterior, Extensor Digitorm Longus, Gastrocnemius and Soleus) from 10 days unilaterally denervated and unilaterally denervated + CNTF exposed rats.

\begin{tabular}{|c|c|c|c|c|c|c|c|c|}
\hline \multirow[b]{2}{*}{ Condition } & \multicolumn{8}{|c|}{ Muscle weight (mg) } \\
\hline & $\mathrm{TA}_{\mathrm{den}}$ & $\mathrm{TA}_{\mathrm{in}}$ & $\mathrm{EDL}_{\text {den }}$ & $\mathrm{EDL}_{\mathrm{in}}$ & $\mathrm{Gas}_{\mathrm{den}}$ & $\mathrm{Gas}_{\text {in }}$ & $\mathrm{Sol}_{\mathrm{den}}$ & $\mathrm{Sol}_{\text {in }}$ \\
\hline Unil. den. & $319 \pm 13$ & $529 \pm 17$ & $111 \pm 4$ & $148 \pm 5$ & $1029 \pm 35$ & $1694 \pm 59$ & $80 \pm 6$ & $144 \pm 4$ \\
\hline Unil. den + CNTF & $305 \pm 9$ & $545 \pm 17$ & $120 \pm 6$ & $159 \pm 6$ & $1047 \pm 36$ & $1795 \pm 71$ & $84 \pm 6$ & $158 \pm 7$ \\
\hline
\end{tabular}

Mean \pm SEM from 21 controls and 12 CNTF treated animals 
could be receptor-mediated or non-specific, as is the case for striatal neurons, which do not express NGF nor CNTF receptors but are protected from degeneration by both trophic factors through an unknown mechanism of action (Kordower et al., 1999). One indication that CNTF may exert a direct action on muscle fibers comes from its effect on the twitch kinetic parameters. The slowing of contraction and half-relaxation times in denervated EDL suggests a direct effect on the muscle, since the nerve was sectioned at the time the pump containing CNTF was implanted. Given that twitch time is also lengthened by CNTF in innervated EDL muscles, we can speculate that this effect also has a direct component over the muscle.

A clear indication of CNTF acting through the nerve comes from our results on the soleus twitch time because this drug significantly increased contraction and relaxation times in innervated muscles and did not affect denervated soleus (Communicated as preliminary results in Ramirez et al. 2001).

With respect to muscle excitability, we detected CNTF-induced sporadic bursts only in innervated muscles. We cannot disregard the possibility that spontaneous, irregular bursts induced by CNTF in denervated muscles could have been masked by fibrillation.

In our experiments, CNTF may have been conveyed to the spinal motoneuron somas by retrograde axonal transport. Retrograde transport of radiolabeled CNTF has been observed in peripheral nerves (Curtis et al., 1993, Mufson, 1999), although its mechanism has not been established (Neet and Campenot, 2001). Furthermore, intramuscular injections of an adenoviral vector coding for a secretable form of CNTF reduced the degeneration of phrenic myelinated nerve fibers in mouse mutants with motoneuronal degeneration (Haase et al., 1999). It is possible then, that the spontaneous electrical activity observed in innervated muscles could be due to an effect of CNTF over motoneurons that indirectly affected muscles. Stoop and Poo (1995) and Liou et al (1997) have shown that CNTF potentiates the spontaneous and the impulse-evoked release of neurotransmitter at developing neuromuscular synapses in Xenopus cell cultures.

Forskolin mimics the effect of CNTF for contraction time only in innervated muscles. Since forskolin increases cAMP levels, the motoneuron mediated effects of CNTF on contraction time could be cAMP-mediated. Because forskolin has no effect on denervated muscles, we can speculate that the direct effects of CNTF over muscle are not mediated by cAMP.

Duration of contraction is a function of temperature, the coupling between the tranverse-tubule dihidropyridin receptors, ryanodin receptors, and the actomyosin ATPase. On the other hand, relaxation time is primarily controlled by the rate of $\mathrm{Ca}^{2+}$ uptake into the sarcoplasmic reticulum. Therefore, with the exemption of temperature, any of these parameters could have been affected by CNTF. Our measurements were done at room temperature, and therefore the twitch kinetics were slowed with respect to measurements done at $37^{\circ} \mathrm{C}$.

\section{Myotrophic role of CNTF on denervated or innervated muscles}

The reports on the protective effects of CNTF on the denervation-induced atrophy and denervated muscle functional performance are contradictory. The dose and type of administration of this cytokine (Kordower et al., 1999), the way results are presented, and the type of muscle studied may contribute in part of the discrepancies. Helgren et al. (1994) reported that CNTF attenuated the soleus weight-loss induced in rats after sectioning the sciatic nerve. In contrast, Martin et al. (1996) reported that CNTF induced a dose-dependent weight loss of innervated gastrocnemius and that the weight of denervated-CNTF exposed muscles was not different from the weight of just denervated muscles. Because Helgren et al. (1994) expressed their results as the ratio of the wet weight of denervated/ innervated muscles that were or were not exposed to CNTF, it is not clear whether the effect of the drug was on the innervated or on the denervated muscle. 
Helgren et al. (1994) also concluded that CNTF protects the soleus from the denervation-induced slowing of the twitch by comparing the difference in the peak contraction times for innervated and denervated muscles that were or were not exposed to CNTF. They reported that for the CNTF treated group, the difference between innervated and denervated muscles was not significant and considered this a manifestation of the CNTF's protective role. We also find a smaller difference for the CNTF treated group as compared to controls, but this is because CNTF causes a slowing of the contraction time for the innervated soleus (see Fig 4A control and CNTF innervated and denervated). Nevertheless, in our experimental conditions we also find, as Helgren's group describes, the protective effect of CNTF over the denervation-induced atrophy for the soleus, quantified as fiber crosssectional area (Ramirez et al. 2000).

We did not find differences in muscle weight between the control and CNTFtreated muscles, whether innervated or denervated. One reason for this may be that we used a lower dose than Helgren et al (1994) or Martin et al. (1996). Different parameters may have differential sensitivities in one organ, and there could also be differential sensitivities between organs and between muscle fiber types. Spontaneous electrical activity has not been recorded by other authors after CNTF treatment, but our studies showed that doses that induced spontaneous discharges in innervated muscles did not modify the wet weight of the same muscles.

It is not easy to compare the effects of CNTF under different dosages and application methods. The range for subcutaneous applications was $1 \mathrm{mg} / \mathrm{Kg}$ daily in rats in the reports of Helgren et al. (1994) and Martin et al. (1996) and of 15$30 \mu \mathrm{g} / \mathrm{Kg}$ every 48 hours in humans (ALS Study Group 1996). For intrathecal delivery, $0.2 \mathrm{mg} /$ day every 48 hours have been given to human patients (Penn et al. 1997). The dose used for the experiments reported here is approximately equivalent to $24 \mathrm{ng} / \mathrm{Kg} /$ day. The reason for delivering CNTF chronically with a pump rather than by daily injections is to avoid animal stress. CNTF stability in osmotic pumps has been demonstrated by Marques and Neto (1997). They reported that $1 / 4$ of CNTF bio-activity is detectable after 7 days of pump implantation in an identical experimental design to the one we used. Moreover, Guillet at al. (1999) detected CNTF in plasma after 14 days of treatment monitored by Elisa and TF 1 cells bioassays. Although we used a very low dose of CNTF, we found notorious effects on excitability and contractile properties of innervated muscles.

Because the use of neurotrophic factors for treatment of neurodegenerative disorders seems promising, it is important that the specific side effects of the cytokines would be characterized. Here we present evidence that in addition to its known action as a neurotrophic factor, CNTF affects skeletal muscle performance in denervated and innervated preparations. In the latter, the increased neuromuscular excitability may be responsible for the cramps and pain described by patients.

\section{ACKNOWLEDGEMENTS}

We want to thank Dr. Daniel Wolff, Dr. Juan Bacigalupo, Dr. Patricio Sáez and Rossana Marsh for their comments on the manuscript. Financed by Fondecyt 1981053 and Dicyt 029743-RU.

\section{REFERENCES}

ALS-CNTF-Treatment Study Group (ACTSG) (1996) A double-blind placebo-controlled clinical trial of subcutaneous recombinant human ciliary neurotrophic factor (rhCNTF) in amyotrophic lateral sclerosis. Neurol 46: 1244-1249

BACHOUD-LEVI A, DEGLON N, NGUYEN J, BLOCH J, BOURDET C, WINKEL L, REMY P, GODDARD M, LEFAUCHEUR JP, BRUGIERE P, BAUDIC S, CESARO P, PESCHANSKI M, AEBISCHER P (2000) Neuroprotective gene therapy for Huntington's disease using a polymer encapsulated BHK cell line engineered to secrete human CNTF. Hum Gene Ther 11: 1723-1729

CURTIS R, ADRYAN KM, ZHU Y, HARKNESS PJ, LINDSAY RM, DISTEFANO PS (1993) Retrograde axonal transport of ciliary neurotrophic factor is increased by peripheral nerve injury. Nature 365 : 253-255 
GUILLET C, AUGUSTE P, MAYO W, KREHER P, GASCAN H (1999) Ciliary neurotrophic factor is a regulator of muscular strength in aging. $J$ Neurosci 19: 1257-1262

HAASE G, PETTMANN B, BORDET T, VILLA P, VIGNE E, SCHMALBRUCH H (1999) Therapeutical benefit of ciliary neurotrophic factor in progressive motor neuronopathy depends on the route of delivery. Ann Neurol 45: 296-304

HELGREN ME, SQUINTO SP, DAVIS HL, PARRY DJ, BOULTON TG, HECK CS, ZHU Y, YANCOPOULUS GD, LINDSAY RM, DISTEFANO PS (1994) Trophic effect of ciliary neurotrophic factor on denervated skeletal muscle. Cell 76: 493-504

HUANG S, WANG F, HONG G, WAN S, KANG H (2002) Protective effects of ciliary neurotrophic factor on denervated skeletal muscle. J Huazhong Univ Sci Technolog Med Sci 22: 148-51

KORDOWER JH, ISACSON O, EMERICH DF (1999) Cellular delivery of trophic factors for the treatment of Huntington's disease: is neuroprotection possible? Exp Neurol 159: 4-20

LIOU JC, YANG RS, FU WM (1997) Regulation of quantal secretion by neurotrophic factors at developing motoneurons in Xenopus cell cultures. J Physiol 503:129-139

MCLENNAN AJ, GASKIN AA, VINSON EN, MARTINEZ LC (1996) Ciliary neurotrophic factor receptor $\alpha$ mRNA in NB41A3 neuroblastoma cells: regulation by cAMP. Eur J Pharmacol 295 : 103-108

MARQUES MJ, NETO HS (1997) Ciliary neurotrophic factor stimulates in vivo myotube formation in mice. Neurosci Letters 234: 43-46

MARTIN D, MERKEL E, TUCKER KK, MCMANAMAN JL, ALBERT D, RELTON J, RUSSEL DA (1996) Cachectic effect of ciliary neurotrophic factor on innervated skeletal muscle. Am J Physiol 271: R14221428
MUFSON EJ, KROIN JS, SENDERA TJ, SOBREVIELA T (1999) Distribution of retrograde transport of trophic factors in the central nervous system: functional implications for the treatment of neurodegenerative diseases. Prog Neurobiol 57: 451-484

NEET KE, CAMPENOT RB (2001) Receptor binding, internalization, and retrograde transport of neurotrophic factors. Cell Mol Life Sci 58: 10211035

PENN RD, JEFFREY MD, KROIN S, YORK MM, CEDARBAUM JM (1997) Intrathecal ciliary neurotrophic factor delivery for treatment of amyotrophic lateral sclerosis (phase I trial). Neurosurgery 40: 94-100

RAMIREZ BU, BAHAMONDE MI, TRONCOSO V, VERGARA C (2000) Ciliary neurotrophic factor affects the expression of the apamin sensitive calcium activated potassium channel in skeletal muscle. Cont Cient Tecnol USACH 123: 31-39

RAMIREZ BU, RETAMAL L, VERGARA C (2001) Trophic capacity of adult motoneurons is modified by CNTF. Biol Res 34 (3-4) R134, Abstract 251

SLEEMAN MW, ANDERSON KD, LAMBERT PD, YANCOPOULOS GD, WIEGAND S J (2000). The ciliary neurotrophic factor and its receptor, CNTFR alpha. Pharm Acta Helv 74: 265-272

STOOP R, POO M (1995) Potentiation of transmitter release by Ciliary Neurotrophic Factor requires somatic signaling. Sci 267: 695-699

WEIS J, LIE DC, RAGOSS U, ZUCHNER SL, SCHRODER JM, KARPATI G, FARRUGGELLA T, STAHL N, YANCOPOULOS GD, DISTEFANO PS (1998) Increased expression of CNTF receptor alpha in denervated human skeletal muscle. J Neuropathol Exp Neurol. 57: 850-857

WINDISCH M, GSCHANES A, HUTTER-PAIER B (1998) Neurotrophic activities and therapeutic experience with a brain derived peptide preparation. J Neural Transm Suppl 53: 289-298 